\title{
Dwarf Galaxies as Low Surface Brightness Galaxies
}

\author{
Evan D. Skillman \\ Astronomy Department, University of Minnesota, \\ Minneapolis, $M N 55455$
}

\begin{abstract}
Because of the weak correlation between surface brightness and luminosity, as galaxy surveys reach to lower surface brightnesses, increasing numbers of dwarf galaxies are found. Thus, studies of dwarf galaxies are relevant to our understanding of the low surface brightness universe. In particular, studies of nearby LSB dwarfs may help to answer questions concerning: (1) star formation in low abundance and low mass surface density envrionments, (2) star formation histories of galaxies and the relative youth of LSB galaxies, and (3) the chemical evolution of LSB galaxies. HST WFPC2 photometry of the resolved stars in nearby dwarf galaxies is particularly useful for all three questions.

$H$ II region abundances remain the best probe of current ISM abundances. Current evidence favors total mass (or luminosity) as the primary factor for determining the present day chemical abundance in both LSB and HSB galaxies. It remains possible that mass surface density (or surface brightness) may be a second controlling parameter.
\end{abstract}

\section{THE IMPORTANCE OF NEARBY DWARF IRREGULARS}

I started to prepare for this talk by reading the Impey \& Bothun (1997; IB97) Annual Review article on LSBs. Their Figures 1 and 2 compare the surface brightness/luminosity $(\mu / \mathrm{L})$ distribution of galaxies from the RC3 with that of galaxies from the APM survey (Impey et al. 1996). As IB97 state, there is a weak correlation between between $\mu$ and $\mathrm{L}$ for the newly discovered galaxies, such that as these surveys probe to fainter and fainter $\mu$, the catalogs begin to be dominated by dwarfs (absolute magnitudes $\geq-18$ ). Thus, it makes sense to pay attention to what has been learned about dwarf galaxies when attempting to understand the LSB galaxy population.

In other words, for important questions like estimating the fraction of $\Omega$ (baryons) or $\Omega$ (mass) that resides in LSB galaxies, large statistical samples are required. However, for certain other questions, detailed studies of prototypes are useful. Obviously, for these prototype studies, the nearest prototype galaxies are the best targets. Studies of these nearby galaxies have the potential to yield insights into the "LSB Universe."

Luckily, there is a great wealth and variety of nearby LSB dwarfs. Mateo (1998) has assembled a marvelous overview of all of the Local Group members. This compilation will be invaluable for future studies and I will take advantage of it right now. Figure 1 shows the $\mu, \mathrm{L}$ distribution (in $\mathrm{V}$ band) for the Local 


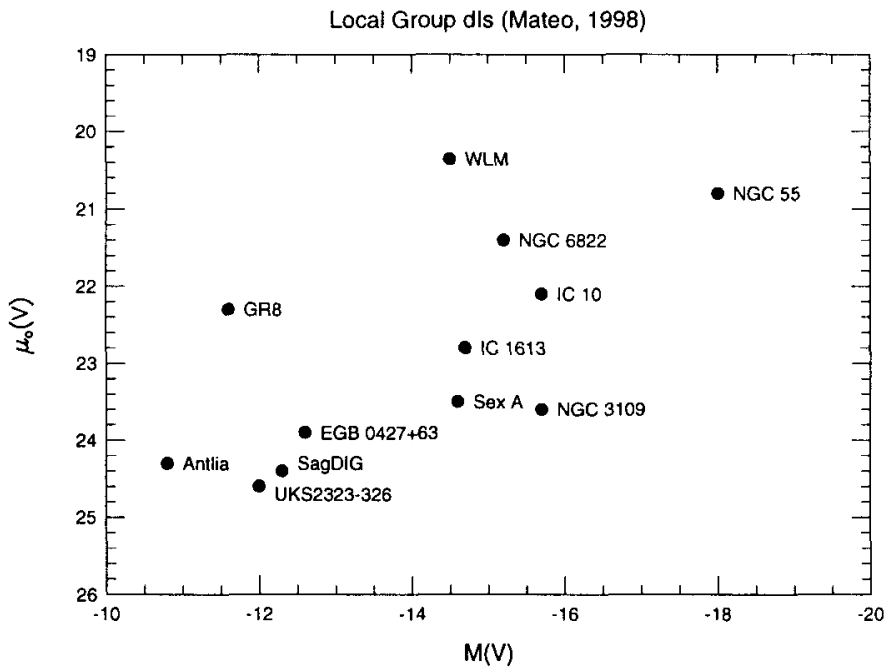

Figure 1. Plot of central surface brightness versus absolute magnitude for several Local Group dwarf irregular galaxies (from Mateo 1998). Note the relative abundance of LSB $(\mu \geq 23)$ systems.

Group dwarf irregular galaxies. Here we see a large diversity in the currently star forming dwarfs, with many galaxies with central surface brightnesses below 23 (the nominal definition for a LSB galaxy). Note that not all Local Group dIs are included (some are still lacking reliable surface brightness measurements!).

These galaxies, and other nearby galaxies like them can be used to answer many questions about LSB galaxies. Here I concentrate on three topics:

- Is star formation different in low surface density and/or low chemical abundance environments? Theoretical calculations indicate that both mass surface density and abundance provide feed back to the star formation process - is there observational evidence for this?

- What are the star formation histories (SFHs) of the LSB dwarfs? Do they experience delayed formation or do their evolution clocks run more slowly (or both)? Is it possible to have galaxy formation occurring in the present epoch?

- Is the chemical evolution of LSB galaxies different from that of HSB galaxies? Is mass surface density a second parameter in determining the present day chemical abundance of galaxies (where total mass is the first parameter)?

\section{STAR FORMATION IN LOW SURFACE DENSITY AND LOW CHEMICAL ABUNDANCE ENVIRONMENTS}

\subsection{Current Star Formation Rates and Thresholds}

Theoretical calculations (e.g., Spaans \& Norman, 1997; Spaans \& Carollo, 1998) and observational evidence (e.g., Israel 1997; Taylor et al. 1998) support the idea that the character of the ISM changes both as a function of decreasing mass surface density and decreasing chemical abundance. 
In the last decade, one idea which has gained considerable attention is that galaxies have star formation thresholds. In his now famous paper, Kennicutt (1989) found that the Toomre disk stability criterion for gravitational instability (Toomre 1964) can be used to predict critical values of the surface gas density $\left(\Sigma_{c}\right)$. These values show a remarkable ability to predict the limits of star formation in the (predominantly high surface brightness) spiral galaxies studied by Kennicutt.

In the last few years, the focus of related research has been to test the theory in different environments, with a particular emphasis on LSB and dwarf galaxies. The following studies all support the threshold theory: (1) van der Hulst et al. (1993) observed a small collection of large LSB spirals. Their HI surface densities were found to lie at or below the critical densities calculated from the Toomre criterion across the extent of their disks. (2) Taylor et al. (1994) observed five HII galaxies. In all cases, the central HI surface densities exceeded $\Sigma_{c}$, and the radius where the HI surface density fell below $\Sigma_{c}$ roughly corresponded to the optical diameter of the galaxy. (This result has been supported by new observations of blue compact galaxies by van Zee et al. 1998). (3) van Zee et al. (1997a) observed ten isolated dwarf galaxies which were divided into LSB dwarf galaxies and "normal" gas-rich dwarf galaxies. Their HI surface densities were found to lie at or below the critical densities. Interestingly, local peaks in the HI surface densities approach the critical value, and these are associated with sites of active star formation as determined from $\mathrm{H} \alpha$ observations.

\subsection{Recent Star Formation Histories}

A tremendous advance in this field has come about due to the fantastic imaging abilities of the Hubble Space Telescope. This has resulted in great improvements in the accuracy of the photometry of resolved stars, and this allows us to produce very detailed recent SFHs for nearby galaxies. As an example, Figure 2 shows two color magnitude diagrams (CMDs) constructed from HST WFPC2 observations. A very important advance is represented by the separation of the "blue plume" into main sequence and blue core helium burning ("blue loop" or HeB) stars.

The blue HeB stars provide a parallel track to the MS in which to observe star formation events. From the number of blue HeB stars, we can calculate the SFR for the age corresponding to this phase of evolution. There are two advantages to using the blue $\mathrm{HeB}$ as an indicator of SFH: (1) The blue HeB stars are about 2 magnitudes brighter than the MS turnoff stars of the same age (e.g., Bertelli et al. 1994). This allows us to probe the recent SFH further back in time (for the same photometric limits); (2) There is little confusion from overlapping generations. All of the blue $\mathrm{HeB}$ stars of a certain magnitude come from the same generation of stars. In practice, the blue HeB stars can probe the SFH back to almost 1 Gyr. At older ages, they blend with the red clump and horizontal branch, becoming degenerate in time.

Figure 3 shows the SFHs for four Local Group dIs constructed from the blue HeB stars. Note the unprecedented time resolution in these SFHs. For this sample, it turns out that the average recent star formation rate correlates with the gas mass content of the galaxies (see Dohm-Palmer et al. 1998). 

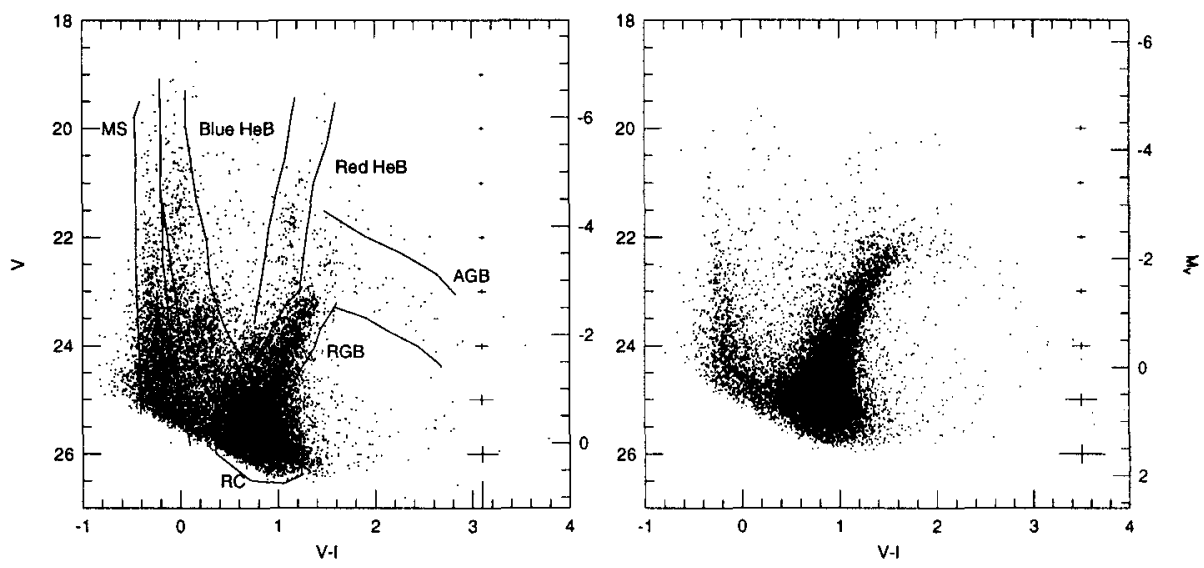

Figure 2. The CMDs in V and I for the Local Group dIs Sextans A (left, from Dohm-Palmer et al. 1997) and the Pegasus dI (right, from Gallagher et al. 1998). Note the paucity of the MS and $\mathrm{HeB}$ populations in Pegasus compared to Sextans A.

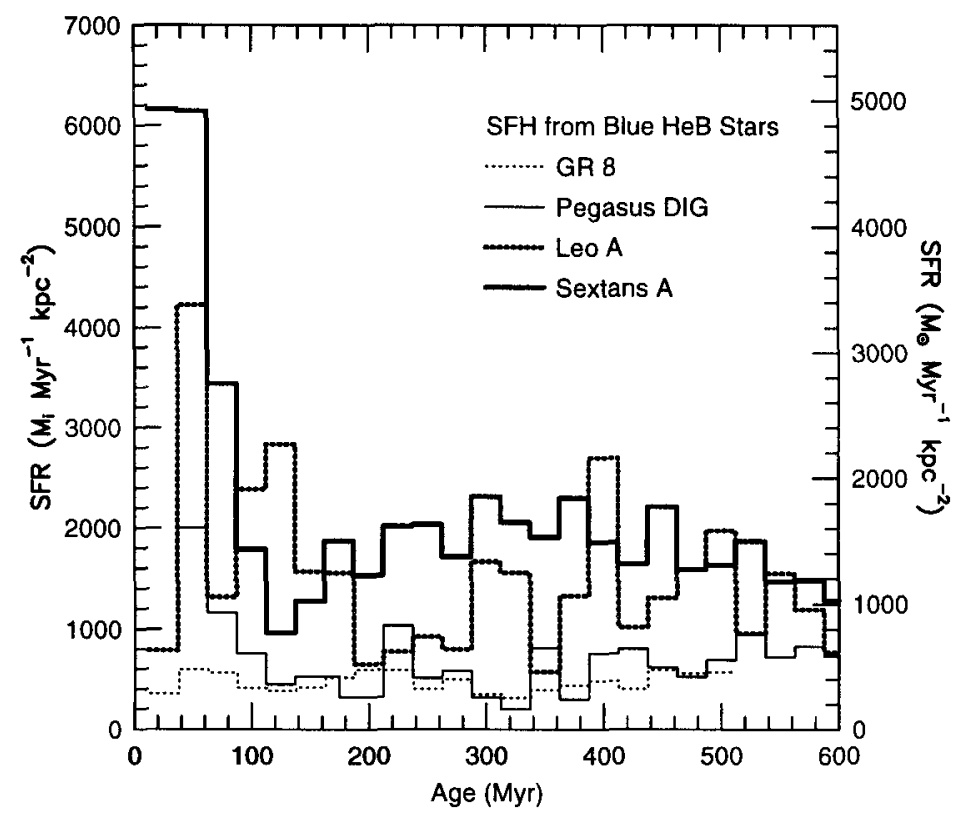

Figure 3. The star formation history of four nearby dI galaxies based on the blue HeB luminosity function. The bins are 25 Myr. Sextans A consistently has the highest SFR/area, followed by Leo A. Pegasus and GR 8 have a very similar SFR/area, which is consistently lower than in the other two galaxies. From Dohm-Palmer et al. 1998. 


\section{THE STAR FORMATION HISTORIES OF NEARBY DWARF IRREGULARS - THE YOUNG GALAXY HYPOTHESIS}

McGaugh \& Bothun (1994) pointed out that the blue colors of LSB galaxies meant that they were inconsistent with "fading" scenarios and hypothesized that LSB galaxies probably formed relatively late and evolved slowly. While more recent surveys (e.g., O'Neil et al. 1997) have shown that LSBs show a large range in color (and therefore evolutionary state), the hypothesis that LSB galaxies may form late and evolve slowly is still of interest (cf. McGaugh \& de Blok 1998). Determining detailed star formation histories (SFH) of galaxies has always been a long-term goal of extragalactic observational astronomy (e.g., Hodge 1989), and measures of the resolved stars in nearby galaxies offer the best hope for very detailed SFHs (e.g., Smecker-Hane et al. 1994). Nonetheless, while $\$ 2.2$ emphasized the successes in modeling the recent SFHs of dIs, constraining the old SFHs of galaxies ( $\geq 1 \mathrm{Gyr}$ ) is a very difficult problem.

For very close galaxies, the SFH can be reconstructed from the MS luminosity function (e.g., Butcher 1977). However, few galaxies are close enough and the old SFHs must come from an understanding of the distribution of stars on the red giant branch. This field has been pioneered with ground-based observations (e.g., Tosi et al. 1991; Greggio et al. 1993; Gallart et al. 1996; Tolstoy \& Saha 1996). Imaging with the WFPC2 on the HST allows vast improvements in the fidelity of the photometry, yielding similar improvements in the observational constraints on the SFHs (e.g., Aparicio et al. 1996).

Recently it has been suggested that the stellar population in the Local Group dwarf irregular Leo A is predominantly young (i.e., stellar ages less than 2 Gyr; Tolstoy et al. 1998). The main diagnostic is the ratio of "red clump" stars (core helium burning stars which overlap the red giant branch in the HR diagram) to red giant branch stars (cf. Bertelli et al. 1992; Gallagher et al. 1998; Tolstoy et al. 1998). Since these stars probe the oldest ages and since the lifetime in the red clump is roughly constant while the lifetime in the red giant branch is a strong function of the mass of the star, this ratio is a relatively robust measure of the average age of the stellar population.

The study of Leo A was based on two orbits of HST observations. Clearly there is potential in this type of study to characterize the SFHs for a large number of nearby galaxies. As the sample increases it will be possible to study SFH (or average stellar age) as a function of both intrinsic galaxy properties (mass, luminosity, surface brightness) and extrinsic galaxy properties (environment, local density). This will allow us to answer the question whether surface mass density and surface brightness correlate with mean stellar age.

\section{ISM ABUNDANCES OF LSB DWARFS}

Surveys of the abundances in a number of H II regions in irregular galaxies show a clear correlation of oxygen abundance with galaxy luminosity (Skillman et al. 1989 and reference therein). (This trend is also seen in spiral galaxies - Zaritsky, Kennicutt \& Huchra 1994 and references therein). However, not all dwarf galaxies comply with the metallicity - luminosity relationship. For example, many blue compact dwarf galaxies (BCDGs), which derive a significant 
Local Group dls (M98) \& LSB dwarts (vZ97) \& LSBs (M94)

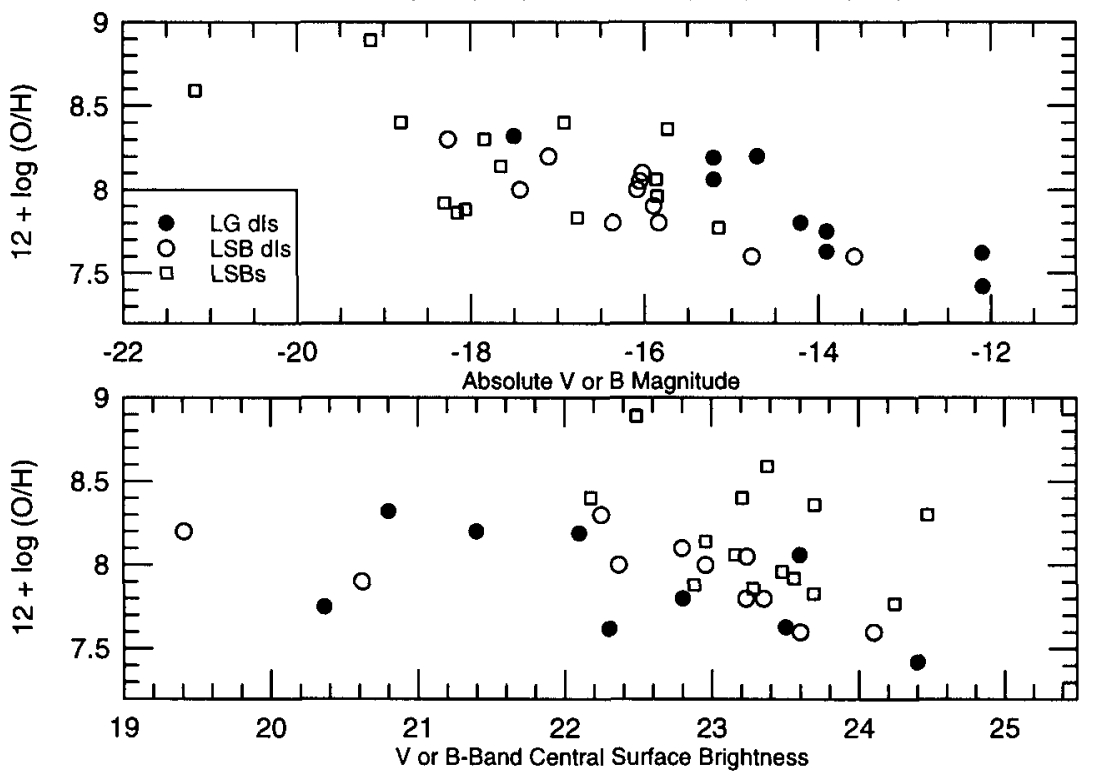

Figure 4. Comparison of HII region oxygen abundance versus absolute magnitude and central surface brightness for Local Group dwarf irregular galaxies from the compilation of Mateo (1998), LSB dwarf irregular galaxies from van Zee et al. (1997), and LSB galaxies from McGaugh (1994).

fraction of their total luminosity from their high surface brightness star forming regions, appear overluminous for their abundance (e.g., Roennback \& Bergvall 1995). If the underlying correlation is between mass and metallicity, then this is expected since the BCDGs have lower M/L ratios. Skillman et al. (1997) have shown that fading also contributes to the scatter in the $\mathrm{O} / \mathrm{H}, \mathrm{L}$ relationship.

On the other hand, the fundamental relationship may not be between mass and metallicity, but between surface density and metallicity. Mould, Kristian, \& DaCosta (1983) first discovered that dwarf elliptical galaxies also show a strong correlation between metallicity and luminosity, and Aaronson (1986) showed that the metallicity vs. luminosity relationships for the two classes of galaxies are roughly identical in both slope and zero-point (although see Mateo (1998) for a more recent review). Since low luminosity, high surface brightness, and relatively metal rich dwarf ellipticals have been observed, Bothun \& Mould (1988) suggested that surface density may be the fundamental parameter determining metallicity (see also Edmunds \& Phillips 1989).

Caldwell et al. (1998) have used the HST to obtain metallicities in two dEs in the M81 group and found that they both comform to the metallicityluminosity relationship as defined by the Local Group dEs, but that the very low central surface brightness object F8D1 lies well off the metallicity - central surface brightness relationship. Thus, they propose that luminosity and not surface brightness is the key parameter determining metallicity. 
This argument is based on a sample of one, and it pertains to the gas poor family of dEs. How does a similar test for gas rich galaxies result? I drew upon data from three different studies in the literature to compose Figure 4. First, I used the compilation of Mateo (1998) to compare Local Group dIs. Then I added the LSB dIs of van Zee et al. $(1997 \mathrm{~b}, \mathrm{c})$, and finally the LSBs studied by McGaugh (1994). While the known correlation in $\mathrm{O} / \mathrm{H}, \mathrm{L}$ is clearly shown, the correlation is much weaker for $\mathrm{O} / \mathrm{H}, \mu$. It would appear that these studies support luminosity as the important parameter.

In the past, LSB galaxies have been characterized as metal poor, with chemical abundances less than one-third of the solar value (McGaugh 1994). A reexamination of all available data indicates that both LSB and HSB galaxies show correlations between metallicity and luminosity, and thus, it is better to characterize LSB galaxies in this way as opposed to all metal poor.

Is this relation universal? Garnett et al. (1997) compared the abundances in different spiral galaxies at similar disk optical surface brightness, and found a clear correlation between the abundance at a given surface brightness and the total luminosity of the galaxy. This suggests that massive spirals are more efficient at enriching their ISM at fixed surface brightness than low mass spirals. (Because of the luminosity - gas mass fraction correlation, this can be cast in terms of simple models tying abundance to gas mass fraction, e.g., McGaugh \& de Blok 1997). Note that the Garnett et al. sample contains a limited range in overall galaxy surface brightness. Adding LSB galaxies to this plot will rovide a strong test (e.g., Pickering 1998), and it will be interesting to see if surface brightness acts as a second parameter in determining present day abundance. This diagram is telling us something fundamental about galaxy evolution. Perhaps we are closer to defining a fundamental set of parameters which govern disk galaxy evolution (e.g., Mollá, Ferini, \& Díaz 1996, and references therein).

Acknowledgments. I would like to thank Liese van Zee for recalculating surface brightness parameters for her LSB dI galaxies so that I could include them in Figure 4. I would also like to thank Chris Taylor and Stacy McGaugh for comments on an earlier draft. Partial support from a NASA LTSARP grant No. NAGW-3189, is gratefully acknowledged.

\section{References}

Aaronson, M. 1986, in Star Forming Dwarf Galaxies and Related Objects, eds. D. Kunth, T.X. Thuan, and J.T.T. Van, Editions Frontieres, 125

Aparicio, A., Gallart, C., Chiosi, C., \& Bertelli, G. 1996, ApJL, 469, 97

Bertelli, G., Bressan, A., Chiosi, C., Fagotto, F. \& Nasi, E. 1994, A\&AS 106, 275

Bertelli, G. Mateo, M., Chiosi, C., \& Bressan, A. 1992, ApJ, 388, 400

Bothun, G. D., \& Mould, J. R. 1988, ApJ, 324, 123

Butcher, H. 1977, ApJ, 216, 372

Caldwell, N., Armandroff, T. E., Da Costa, G. S., \& Seitzer, P. 1998, AJ, 115, 535

Dohm-Palmer, R. C., Skillman, E. D., Saha, A., Tolstoy, E., Mateo, M., Gallagher, J., Hoessel, J., \& Dufour, R. J. 1997, AJ, 114, 2527

Dohm-Palmer, R.C., Skillman, E.D., Gallagher, J., Tolstoy, E., Mateo, M., Dufour, R.J., Saha, A., Hoessel, J., \& Chiosi, C. 1998, AJ, 116, 1227 
Edmunds, M. G., \& Phillips, S. 1989, MNRAS, 241, 9p

Gallagher, J. S., Tolstoy, E., Dohm-Palmer, R. C., Skillman, E. D., Cole, A., Hoessel, J., Saha, A., \& Mateo, M. 1998, AJ, 115, 1869

Gallart, C., Aparicio, A., \& Víchez, J. M. 1996, AJ, 112, 1950

Garnett, D. R., Shields, G. A., Skillman, E. D., Sagan, S. P., \& Dufour, R. J. 1997, ApJ, 489, 63

Greggio, L., Marconi, G., Tosi, M., \& Focardi, P. 1993, AJ, 105, 894

Hodge, P.W. 1989, ARA\&A, 27, 139

Impey, C., \& Bothun, G. 1997, ARA\&A, 35, 267

Impey, C. D., Sprayberry, D., Irwin, M. J., \& Bothun, G. D. 1996, ApJS, 105, 209

Israel, F. P. 1997, A\&A, 328, 471

Kennicutt, R. C., Jr. 1989, ApJ, 344, 171

Mateo, M. 1998, ARA\&A, 36, in press

McGaugh, S. S. 1994, ApJ, 426, 135

McGaugh, S. S., \& Bothun, G. D. 1994, AJ, 107, 530

McGaugh, S. S., \& de Blok, W. J. G. 1997, ApJ, 481, 689

McGaugh, S. S., \& de Blok, W. J. G. 1998, ApJ, 499, 41

Mollá, M., Ferini, F., \& Díaz, A. I. 1996, ApJ, 466, 668

Mould, J. R., Kristian, J., and Da Costa, G. S. 1983, ApJ, 270, 471

O'Neil, K., Bothun, G. D., Schombert, J., Cornell, M. E., \& Impey, C. D. 1997, AJ, 114,2448

Pickering, T. E. 1998, PhD. Thesis, University of Arizona

Roennback, J., \& Bergvall, N. 1995, A\&A, 302, 353

Skillman, E. D., Bomans, D. J., \& Kobulnicky, H. A. 1997, ApJ, 474, 205

Skillman, E. D., Kennicutt, R. C. \& Hodge, P. W. 1989, ApJ, 347, 875

Smecker-Hane, T. A., Stetson, P. B., Hesser, J. E., \& Lehnert, M.D. 1994, AJ, 108, 507

Spaans, M., \& Carollo, C. M. 1998, ApJ, 502, 640

Spaans, M., \& Norman, C. A. 1997, ApJ, 483, 87

Taylor, C. L., Brinks, E., Pogge, R. W., \& Skillman, E. D. 1994, AJ, 107, 971

Taylor, C. L., Kobulnicky, H. A., \& Skillman, E. D. 1998, AJ, in press

Tolstoy, E., Gallagher, J. S., Hoessel, J., Saha, A., Skillman, E. D., Dohm-Palmer, R. C., \& Mateo, M. 1998, AJ, 116, 1244

Tolstoy, E., \& Saha, A. 1996, ApJ, 462, 672

Toomre, A., 1964, ApJ, 139, 1217

Tosi, M. Greggio, L., Marconi, G., \& Focardi, P. 1991, AJ, 102, 951

van der Hulst, J. M., Skillman, E. D., Smith, T. R., Bothun, G. D., McGaugh, S. S., \& de Blok, W. J. G. 1993, AJ, 106, 548

van Zee, L., Haynes, M. P., Salzer, J. J., \& Broeils, A. H. 1997a, AJ, 113, 1618

van Zee, L., Haynes, M. P., \& Salzer, J. J. 1997b, AJ, 114, 2479

van Zee, L., Haynes, M. P., \& Salzer, J. J. 1997c, AJ, 114, 2497

van Zee, L., Skillman, E. D., \& Salzer, J. J. 1998, AJ, 116, 1186

Zaritsky, D., Kennicutt, R. C., \& Huchra, J. P. 1994, ApJ, 420, 87 